Published in final edited form as:

J Acquir Immune Defic Syndr. 2017 February 01; 74(Suppl 2): S88-S95. doi:10.1097/QAI.

0000000000001234.

\title{
Longitudinal Trajectories of HIV Care Retention in North Carolina
}

Kimberly A. Powers, MSPH, PhD ${ }^{1}$, Erika Samoff, PhD $^{2}$, Mark A. Weaver, PhD ${ }^{3,4}$, Lynne A. Sampson, MPH, $\mathbf{P h D}^{2,3}$, William C. Miller, MD, PhD, $\mathbf{M P H}^{5}$, Peter A. Leone, $\mathbf{M D}^{3}$, and Heidi Swygard, MD, MPH ${ }^{2,3}$

${ }^{1}$ Department of Epidemiology, Gillings School of Global Public Health, University of North Carolina, Chapel Hill, NC, USA

${ }^{2}$ Communicable Disease Branch, Division of Public Health, North Carolina Department of Health and Human Services, Raleigh, NC, USA

${ }^{3}$ Department of Medicine, School of Medicine, University of North Carolina, Chapel Hill, NC, USA

${ }^{4}$ Department of Biostatistics, Gillings School of Global Public Health, University of North Carolina, Chapel Hill, NC, USA

${ }^{5}$ Division of Epidemiology, College of Public Health, The Ohio State University, Columbus, $\mathrm{OH}$, USA

\section{Abstract}

Background-Long-term HIV care and treatment engagement is required for maximal clinical and prevention benefits, but longitudinal care patterns are poorly understood. We used the last ten years' worth of HIV surveillance data from North Carolina (NC) to describe longitudinal HIV care trajectories from diagnosis.

Methods-We conducted a retrospective, population-based cohort study of all persons newly HIV-diagnosed in NC between March 31, 2006 and March 31, 2015 (N=16,207). We defined HIV care attendance in each three-month and six-month interval after diagnosis as the presence of viral load and/or CD4 records (care visit proxies) in the interval. We used group-based trajectory modeling to identify common care trajectories and baseline predictors thereof.

Results-A predicted $26 \%$ of newly HIV-diagnosed person showed consistently high care attendance over time; $~ 16 \%$ exhibited steadily declining attendance; $26 \%$ showed consistently low attendance; $\sim 17 \%$ had initially weak attendance with an increase starting $\sim 1.5$ year later; and $\sim 15 \%$ showed initially weak attendance with an increase starting $\sim 3$ years later. Older age at diagnosis was protective against all sub-optimal trajectories (with the "consistently high" pattern as referent), and MSM status was protective against three of the four sub-optimal patterns.

Contact information for correspondence and reprints: Kimberly A. Powers, MSPH, PhD, Department of Epidemiology, CB 7435, Gillings School of Global Public Health, The University of North Carolina at Chapel Hill, Chapel Hill, NC 27599-7435 USA, T: +1 919-962-0076; F: +1 919-966-2089, powersk@email.unc.edu.

Previous presentations: Portions of this work were presented at the HIV Research for Prevention conference, Chicago, IL, October 17-21, 2016.

Conflicts of Interest: We have no conflicts of interest to declare. 
Conclusions-As measured by surveillance-based laboratory proxies, most newly HIVdiagnosed persons exhibited sub-optimal care trajectories, but there was wide variation in the particular pathways followed. The insights provided by this analytical approach can help to inform the design of epidemic models and tailored interventions, with the ultimate goal of improving HIV care engagement and transmission prevention.

\section{Keywords}

HIV care; continuum; cascade; retention; engagement; surveillance

\section{Introduction}

Antiretroviral therapy (ART) for people living with HIV/AIDS (PLWHA) dramatically improves health ${ }^{1}$ and reduces onward HIV transmission. ${ }^{1,2}$ Unfortunately, a minority of PLWHA in the United States (US) attains the levels of HIV care engagement and successful treatment required to realize these benefits, with only $30 \%-40 \%$ being retained in care and $19 \%-30 \%$ achieving viral suppression. ${ }^{3-6}$ Recognition of these systemic failures along the "HIV care continuum" has led to strong research and programmatic emphases on understanding and improving HIV-infected persons' engagement with care and treatment, such that the individual-level benefits of ART can be translated more effectively to the population level.

Despite the intensive efforts directed in recent years toward measuring and improving HIV care engagement and retention, the longitudinal pathways that HIV-infected persons follow through the HIV care system are poorly understood. Indeed, while the term "continuum" acknowledges that movement from HIV diagnosis to viral suppression is often non-linear, many continuum analyses are cross-sectional and the particular routes that HIV-infected persons do in fact follow are not well-defined. Insights about longitudinal trajectories of HIV care engagement could provide useful information for designing and targeting interventions to particular groups at crucial times. ${ }^{7}$ For example, if we could distinguish people at diagnosis who are likely to consistently attend HIV care visits over the long term from those who are likely to initially attend but subsequently trail off, we could identify subsets of PLWHA in whom greater support is warranted from their very first contact with the healthcare system. Identification of such care engagement pathways could also inform the structure of mathematical models describing the HIV care continuum and its effects on population-level HIV incidence.

In this study, we used the last ten years' worth of HIV surveillance data from North Carolina (NC) to describe longitudinal HIV care trajectories followed by HIV-infected persons from the time of HIV diagnosis, and to assess demographic and clinical predictors of each HIV care trajectory in this population. In addition to seeking information for designing and targeting intervention strategies in $\mathrm{NC}$, our more general intention was to provide a case study illustrating the use of powerful analytical methods that can be readily adapted to HIV care datasets in other settings. 


\section{Methods}

\section{Study design, setting, and population}

We conducted a retrospective cohort study of persons becoming newly HIV-diagnosed in North Carolina over the last ten years. Eligible diagnoses were those occurring between March 31, 2006 and March 31, 2015 (inclusive), ensuring that all persons could contribute at least one year of longitudinal data prior to the close of data collection on March 31, 2016.

\section{Data source}

All study data were drawn from the North Carolina enhanced HIV/AIDS Reporting System (eHARS), an electronic surveillance system storing all new HIV and AIDS diagnoses made by physicians, hospitals, and laboratories in NC since 1982. As of July 1, 2013, reporting to eHARS of all CD4 and viral load tests performed in the state has been mandated by law. Prior to this date, the reporting mandate applied only to detectable viral loads and CD4 counts < 200; however, many reporting entities transmitted all viral load and CD4 results before the mandate required them, rather than filtering out the undetectable viral loads and CD4 counts >200 that were not subject to the mandate. Limited sociodemographic data (age, race, sex, and transmission risk group) collected at the time of diagnosis are also housed in eHARS. Dates of death identified in annual searches of vital registries are entered into eHARS for those who die.

\section{Outcome definitions - HIV care}

Based on recommendations of several major medical and public health organizations that HIV-infected persons should attend HIV primary care visits every three to six months, ${ }^{8-11}$ we specified two main HIV care outcome measures to encompass this full range: one that defines care attendance using a three-month interval and a separate one that uses a six-month interval. For each outcome, we assessed HIV care trajectories based on a dichotomous determination as to whether $\geq 1$ HIV primary care visit occurred for a given participant during the specified interval ( 3 or 6 months), starting at the first such interval after diagnosis. As is standard with surveillance-based assessments of HIV care engagement, ${ }^{12}$ we used the presence of a CD4 or viral load result in the surveillance dataset as a proxy for an HIV primary care visit. Although imperfect, these laboratory measures have been found to perform reasonably well as proxies for routine care in clinical cohort data. ${ }^{13}$

Despite anecdotal evidence suggesting that some clinicians are comfortable scheduling visits less frequently than every six months in patients with well-controlled viremia, we did not additionally analyze HIV care engagement using a one-year interval for two reasons: 1) treatment guidelines ${ }^{14}$ continue to recommend quarterly to semi-annual frequency of the laboratory tests serving as our care engagement proxy, and 2) annual data points would result in less granularity for discriminating separate care trajectories. For this latter reason, we also chose not to assess surveillance-based proxies of the care retention indicators recommended by the Department of Health and Human Services (DHHS) or the Institute of Medicine (IOM), both of which classify patient care status annually based on frequency of and spacing between visits within a given year. ${ }^{8,10}$ 


\section{Statistical Analyses}

We used group-based trajectory modeling, ${ }^{15}$ which is similar to latent class analysis, to identify clusters (i.e., "trajectory groups") of newly HIV-diagnosed persons with similar patterns of HIV care attendance. With this approach, which was first developed in the field of developmental psychology, ${ }^{15}$ we assume that the population comprises a mixture of distinct groups defined by their longitudinal patterns in the outcome of interest (here, HIV care attendance). The approach uses flexible polynomial functions fitted to individual care attendance events over time, and allows prediction of each participant's probability of membership in each identified "trajectory group," as well as estimation of the overall proportions of participants in each group. Applications of this approach to the study of other longitudinal behavioral patterns have included analyses of physical aggression, ${ }^{15}$ anxiety, ${ }^{16}$ substance abuse, ${ }^{17}$ and adherence to HIV prevention products. ${ }^{18}$

In addition to our primary cohort (i.e., persons diagnosed between March 31, 2006 and March 31, 2015), we also conducted trajectory analyses in the subset of persons diagnosed between July 1, 2013 and March 31, 2015 (i.e., only those persons diagnosed after reporting of all CD4 and viral load results became mandatory) to assess whether changes in reporting rules would alter the care patterns observed and conclusions drawn.

We defined time zero as the date of HIV diagnosis. Participants were censored at the time of death (where applicable) or at the maximum amount of potential follow-up, given the timing of diagnosis vis-à-vis administrative end. For example, a person diagnosed on March 31, 2014 was censored after the interval that ended two years post-diagnosis (i.e., after the fourth interval in the analysis of six-month outcomes and after the eight interval in the analysis of three-month outcomes), as data were only collected through March 31, 2016 in our dataset. Persons dying before the end of the first outcome interval (3 or 6 months) were excluded from the analysis.

For each outcome interval (three-month or six-month) and cohort (full or subset), we tested predictor-free trajectory models that specified the existence of two, three, four, or five trajectory groups, using the Bayesian information criterion (BIC) to identify the best-fitting number of trajectories. We decided a priori not to consider models with higher numbers of trajectory groups to ensure that model results would be simple enough to be easily interpretable. Given the dichotomous nature of the outcome (a visit did or did not occur within a given interval), we specified a logit link and binomial distribution in each model. To allow maximum flexibility in trajectory forms, we fit cubic polynomials. To avoid undue influence from smaller numbers of observations at the longest follow-up times, we assessed trajectories over the first eight years (rather than the maximum possible ten years) after diagnosis in the full cohort, and over the first 2.5 years (rather than the maximum possible 2.75 years) in the more recent subset.

After selecting the number of trajectories with the predictor-free models, we then assessed several potential predictors of membership in each trajectory group. The predictors available in eHARS that we assessed were sex (male vs. female), race/ethnicity (dichotomized as white non-Hispanic vs. all other categories), transmission risk group (dichotomized as men who have sex with men vs. all others), age at diagnosis (specified as a continuous variable), 
and first CD4 count at care entry (specified as an ordinal variable with a value of 1 for $\geq 500$, 2 for $350-499,3$ for $200-349$, and 4 for $<200$ ). We conducted the predictor assessment using a two-stage process. ${ }^{19}$ First, we constructed multinomial logistic regression models in which the explanatory variables were the potential predictors and the outcome was the trajectory group assigned to each person based on his/her maximum posterior membership probability in the final predictor-free model (described above). Second, we constructed a new groupbased trajectory model for each cohort (full or subset) and interval (three-month or sixmonth) combination, specifying the number of groups identified in the predictor-free model selection process and including the predictors found to be significant at alpha $=0.20 \mathrm{for}$ at least one trajectory group comparison in the corresponding multinomial logistic regression model of stage one. The inclusion of this second step ensures that predictor coefficient estimates and corresponding confidence intervals properly account for the probabilities of membership in each group and covariance between parameter estimates.

As a sensitivity analysis to complement the predictor-free "recent subset" analysis, we also analyzed trajectories over the first 2.5 years after diagnosis only among persons diagnosed in the first 2.75 years of the study period (i.e., between March 31, 2006 and December 31, 2008). This analysis allows us to compare the shorter-term trends seen in the "recent subset" (i.e. late subset) against the patterns seen over the same 2.5-year window after diagnosis in a sub-cohort diagnosed early in the study period.

All analyses were conducted using SAS software, version 9.4 (@ 2013, SAS Institute, Inc., Cary, NC, USA).

\section{Results}

A total of 16,207 people were newly diagnosed with HIV in NC between March 31, 2006 and March 31, 2015. Of these, 15,887 (98.0\%) and 15,784 (97.3\%) survived long enough (i.e., through the first interval) to be included in the full cohort analysis using the threemonth and six-month outcome intervals, respectively (Table 1). Members of the study population were relatively young (median age at diagnosis 34 years), most (63\%) were black/non-Hispanic, and of those with risk group information available ( 62\%), most (79\%) were men who have sex with men. The subset of people diagnosed from July 1, 2013 through March 31, 2015 tended to be younger (median age at diagnosis 31 years) and were more likely to be MSM ( $84 \%$ of those with data available), but the racial/ethnic distribution was similar to that of the full cohort.

Overall, an average of about $28 \%$ of those in the full cohort attended an HIV care visit in a given 3-month interval and about $42 \%$ attended in a given six-month interval (Figure 1) over most of the maximum eight-year follow-up period, with higher attendance ( $60 \%$ and $\sim 67 \%$, respectively) in the first interval. In the subset of more recent diagnoses, approximately $78 \%$ and $84 \%$ attended a visit in the first three and six months, respectively. The proportion attending visits in a given quarterly or semi-annual interval in this subgroup then declined to about $35 \%$ and $60 \%$, respectively, by 2.5 years. 
In our predictor-free model selection process for each outcome interval in the full cohort, we determined the optimal number of trajectories to be five. Patterns of care attendance according to a five-trajectory model were qualitatively similar across the three-month and six-month outcome definitions (Figure 2, panels A and B). Specifically, a predicted $26 \%$ of newly diagnosed persons showed consistently high care attendance ( $\sim 60 \%$ or $\sim 85 \%$ average probability of attending a visit in each three-month or six-month interval, respectively); about $16 \%$ exhibited steadily declining HIV care attendance (falling from $\sim 65 \%-75 \%$ attendance at the first interval to $15 \%-25 \%$ attendance by year eight); about $26 \%$ exhibited a consistent pattern of very low attendance $(<15 \%)$ after possibly attending the initial visit; approximately $17 \%$ had weak attendance at the very beginning but began to show an increase in attendance from $~ 1$ year after diagnosis; and about $15 \%$ showed initially weak attendance with a steady increase starting $\sim 3$ years after diagnosis. We subjectively labeled these groups "consistently high," "steadily declining," "consistently low," "early increasing," and "late increasing," respectively.

In the subset analysis restricted to those persons diagnosed after reporting of all CD4 and viral load results became mandatory (i.e., from July 1, 2013 onward), only the models based on the three-month outcome converged. In this model, five groups emerged (Figure 2C) that were qualitatively similar to those observed in the full cohort, although the predicted proportions of persons in the "early increasing" (3\%) and "consistently low" (15\%) groups were considerably smaller than they were in the full cohort. Additionally, the predicted size of the "steadily declining" trajectory group (42\%) was considerably larger. We note that because we performed the model selection process separately for each cohort (full or subset) and outcome interval (quarterly or semiannual), and because the specification of cubic polynomials allows trajectory shapes to vary across trajectory groups, our approach was capable of detecting highly variable trajectory patterns across cohort/interval combinations if present. (In other words, the similarity of results across the different populations and intervals is not simply an artifact of the methods.) In our sensitivity analysis among those diagnosed in the first 2.75 years of the study period, we again identified five trajectories (Supplemental Figure). In this early sub-cohort, the "consistently low" group was considerably larger (43.6\%) than in the more recent subset, the "steadily declining" group was smaller (8.7\%), and the "early increasing" group was larger (12.1\%) but exhibited only a transient and much less pronounced increase in care attendance.

In stage one of our predictor analyses in the full cohort, all five predictors (age at diagnosis, sex, race/ethnicity, risk group, and CD4 at care entry) were identified with both the threemonth and six-month outcome intervals for further examination in stage two. All predictors but race were identified for inclusion in the stage two predictor analyses in the more recent subset. In stage two, the subset model did not converge with predictors included, but in the full cohort, we found increasing age at diagnosis to be consistently protective against all suboptimal trajectories when compared to the "consistently high" group (Table 2): odds ratios (ORs) ranged across sub-optimal trajectory groups and outcome intervals from 0.81 (95\% CI: $0.78,0.83)$ to $0.88(0.85,0.91)$ for each five-year age increase. Male sex was associated with the "consistently low" pathway (versus "consistently high") based on both outcome intervals, but was not strongly associated with the other sub-optimal trajectories. White, nonHispanic race/ethnicity was also associated with the "consistently low" trajectory across 
both outcome intervals, along with the "steadily declining" pattern. MSM status appeared to be protective against the three least optimal trajectories (i.e., "consistently low," "steadily declining," and "late increasing"). In general, lower CD4 counts were associated with slightly lower odds of membership in the "steadily declining," "early increasing," and "late increasing" trajectory groups.

We a priori chose to examine models with no more than five care trajectory groups, but because the five-group models were consistently preferred on the basis of the BIC, we conducted a post-hoc analysis to explore whether inclusion of six-group models would have modified our interpretations. With both quarterly and semiannual intervals in the full cohort, six groups were preferred according to the BIC, but the corresponding model essentially split the "early increasing" and "late increasing" trajectories into a third "intermediate increasing" group comprising only $~ 5 \%$ of the population (results not shown), so the main insights arising from this modeling approach remained largely unchanged. Six-group models did not converge for either outcome interval in the subset of more recent diagnoses.

\section{Discussion}

Through the application of group-based trajectory modeling to a decade's worth of HIV surveillance data, we have identified a small set of HIV care trajectories that PLWHA appear to follow from the time of diagnosis in North Carolina. Results were qualitatively similar whether we used a care attendance interval of three or six months. For both outcome intervals we observed five broad patterns: "consistently high," "consistently low," "steadily declining," "early increase," and "late increase." The predicted proportions of the population exhibiting each of these patterns was similar across outcome intervals. However, the estimated proportions attending a visit at a given time point in a given trajectory were generally higher with the six-month interval than with the three-month interval, as attendance every six months is clearly less difficult to achieve.

No single "gold standard" care attendance interval applies to all persons over the entire duration of infection. Most guidelines suggest visits every three to six months; ${ }^{8-11}$ some recommendations suggest that visits may be moved from a three-month to a six-month interval once a patient has achieved stable viral suppression. ${ }^{11}$ We also note that although some physicians may space visits at even greater intervals in patients with controlled viremia, treatment guidelines ${ }^{14}$ continue to recommend quarterly to semi-annual performance of the laboratory tests defining our outcome. The combined results from our three-month and six-month analyses may thus be thought of as providing lower and upper bounds on the extent to which "acceptable" levels of care engagement are achieved according to time since HIV diagnosis, with the relevance of the three-month vs. six-month results varying across populations. In cohorts with large proportions of PLWHA on successful ART, the care trajectories based on six-month intervals may be the more relevant patterns, whereas trajectories based on three-month intervals may be more relevant in populations with less well-established ART use.

These types of analyses can complement more traditional "continuum" estimation by identifying time trends in HIV care patterns that would be obscured with typical cross- 
sectional approaches or even longitudinal analyses (e.g., those shown for illustration in Figure 1) that do not allow for heterogeneity in engagement patterns. Our results suggest, for example, that moderate but steadily declining HIV care attendance over the first two years is likely to continue on a downward trajectory, but that very low attendance from the first year after diagnosis may improve. The observed patterns underscore that while a strong public health emphasis on initial linkage to care is important, the resources committed to those efforts need to be balanced with retention maintenance and care re-engagement efforts over the long term. More generally, insights about the likely range of future time trends given an observed pattern to date can inform the timing of care engagement interventions, such that they are targeted to the right people at the right times.

The baseline predictors that we found to be associated with sub-optimal HIV care trajectories are consistent with results of previous cross-sectional analyses of HIV care engagement, ${ }^{20-23}$ providing additional evidence that persons characterized by these predictors may need improved support. In particular, younger age, non-white race/ethnicity, higher CD4 count, and transmission risk group other than MSM were associated to at least some extent with at least some of the sub-optimal trajectories. The use of group-based trajectory modeling enables more nuanced assessment of these predictors than more traditional cross-sectional approaches, however, by recognizing substantively different patterns of "sub-optimal" care with which a given covariate may be associated. We note that in this particular analysis, many of the predictor associations were not especially strong, suggesting that baseline prediction of likely trajectories - at least in the context of limited predictor information in surveillance data - could be challenging in practice. We also note that because we dichotomized transmission risk group and race/ethnicity for simplicity and ease of interpretation, any important trajectory differences between (for example) Hispanics and non-Hispanics, or between people who inject drugs and people who do not inject drugs, were not discernable in our analyses. Additionally, the predictor analyses may have been biased by the exclusion of a sizeable proportion of our cohort with missing transmission risk group information, although the direction and magnitude of such a bias are difficult to predict.

In addition to potentially allowing identification of people who are likely to follow suboptimal trajectories, as well as identification of key time points at which trajectories diverge, the results of these analyses may also be useful in the development of HIV epidemic models. These models have become increasingly complex in the "Treatment as Prevention" era: because ART receipt depends strongly on HIV diagnosis and care, mathematical models have had to incorporate these features. However, the optimal way in which to include care entry, disengagement, and re-engagement in transmission models remains an open question. Trajectory analyses such as ours can help to identify care attendance "phenotypes" (e.g., "consistently high" and "consistently low" attenders) that can then be explicitly built into the model structure.

It is important to note that the trajectory groups we identified and the corresponding shapes of their HIV care patterns are not fixed entities to which HIV-infected persons "belong." Furthermore, we do not mean to imply that any two persons follow exactly the same trajectory. Instead, our approach enables objective summarization and reduction of complex 
longitudinal data to a simple form, allowing a comprehensible, approximate description of complex realities that can inform our approach to improving these realities. We also note that while the specific patterns we identified might be broadly generalizable to similar settings in the southeastern United States with heavy HIV burdens, different patterns of care attendance might be observed in other types of settings.

The surveillance data on which our analyses were based have limitations, as we $\mathrm{e}^{12,24}$ and others ${ }^{13,25}$ have described previously. These data do not allow us to easily discern people who discontinue care from those who move to a different state, as both outcomes will be characterized in the database only by the absence of CD4/VL results. This limitation is likely to result in underestimated HIV care engagement, although the magnitude of any such bias and the extent to which it might affect different trajectory patterns is difficult to estimate. Additionally, although surveillance data on CD4 and viral load have tremendous utility in population-based continuum estimation, they are imperfect proxies for primary HIV care visits, ${ }^{12,13}$ the true outcome of interest. We also note that only a very few sociodemographic and clinical variables are contained in the surveillance data source, limiting our ability to richly characterize predictors of care engagement trajectories in this study.

As we have noted, reporting rules in North Carolina have changed over time, with mandates for full reporting of all CD4 and viral load values coming only in July of 2013. To account for this aspect of our data source, we conducted trajectory analyses in both the full cohort and in the subset of cohort members diagnosed from July 1, 2013 onward. The results of the subset analyses were similar to those observed in the full cohort; however, the "early increase" and "late increase" trajectories emerged and peaked earlier in the subset analysis. Additionally, the "early increase" and "consistently low" groups were considerably smaller in the subset of more recent diagnoses than in the whole cohort, and the "steadily declining" group was considerably larger. We found similar differences between the recent subset trajectory group sizes and those among an "early" cohort in supplemental sensitivity analyses. The overall proportions attending HIV care in each interval were also higher in the recent subset than in the full cohort (as illustrated in Figure 1), suggesting that increased capture by surveillance of CD4 and viral load results in recent years may well play a role in the differences between the two main analysis sets. Some differences may also be due to true changes in care attendance trends as the ART era has matured. For example, the second-year decline in overall attendance in the recent subset according to the three-month but not the six-month interval (Figure 1) suggests that in recent years, clinicians and/or patients may be intentionally spacing scheduled visits farther apart once care has been well-established.

Future applications of these methods to clinical cohort data with richer covariate information may allow fuller, more nuanced assessment of predictors. However, clinical cohort data generally include information starting only at the time of initial care entry, which can occur years after diagnosis and thus does not allow a full picture of care trajectories over time. Additionally, as with surveillance data, it is generally not possible in analyses of clinical cohort data to determine whether a person who appears to be out of care is truly out of care, or rather in care elsewhere (but invisible to the data system). Optimal assessment of longitudinal trajectories in PLWHA will thus require novel methods of linking data across 
data systems, and/or active enrollment and prospective follow-up of population-based cohorts, such that care engagement from diagnosis can be accurately monitored regardless of patient migration or transfers.

Ideally, future analyses will also include time-varying predictors to enable identification of specific events or other triggers that might differentially affect one trajectory group versus another. ${ }^{26}$ Extensions of trajectory modeling applications to include dual-trajectory modeling 15 of both HIV care engagement and viral suppression, or care engagement and transmission risk behaviors, could also prove useful. These types of analyses, especially if combined with dynamic transmission models, could provide powerful quantitative information about the transmission implications of different care trajectory patterns and the corresponding range of interventions that could be applied.

In conclusion, group-based trajectory modeling is a promising approach for understanding longitudinal HIV care patterns, identifying characteristics that can predict the patterns that particular sub-populations are likely to follow, and specifying key time points at which care trajectories are likely to diverge. This analytical approach can complement more traditional cross-sectional analyses of the HIV care continuum by elucidating the ways in which care engagement changes over the course of infection and allowing for heterogeneity in these longitudinal care engagement patterns. The insights provided by these analyses can help to inform the design of HIV epidemic models and the targeting and timing of tailored interventions, with the ultimate goal of increasing care engagement to maximize the clinical and preventive benefits of ART.

\section{Supplementary Material}

Refer to Web version on PubMed Central for supplementary material.

\section{Acknowledgments}

We thank John Barnhart and Jason Maxwell at the North Carolina Division of Public Health for their generous provision of time and assistance with the NC eHARS data.

The eHARS data used in this study were provided by The NC Department of Health and Human Services, Division of Public Health (DPH). The NC DPH does not take responsibility for the scientific validity or accuracy of methodology, results, statistical analyses, or conclusions presented.

Sources of Funding: This work was supported by the National Institutes of Health (grant numbers KL2 TR001109, R03 MH100987, UL1 TR001111) and an administrative supplement to the UNC Center for AIDS Research, an NIH-funded program (P30 AI50410).

\section{References}

1. Cohen MS, Chen YQ, McCauley M, et al. Prevention of HIV-1 infection with early antiretroviral therapy. N Engl J Med. 2011; 365(6):493-505. [PubMed: 21767103]

2. Rodger AJ, Cambiano V, Bruun T, et al. Sexual Activity Without Condoms and Risk of HIV Transmission in Serodifferent Couples When the HIV-Positive Partner Is Using Suppressive Antiretroviral Therapy. JAMA. 2016; 316(2):171-81. [PubMed: 27404185]

3. Gardner EM, McLees MP, Steiner JF, et al. The spectrum of engagement in HIV care and its relevance to test-and-treat strategies for prevention of HIV infection. Clin Infect Dis. 2011; 52(6): 793-800. [PubMed: 21367734] 
4. Greenberg AE, Hader SL, Masur H, et al. Fighting HIV/AIDS in Washington, D.C. Health Aff (Millwood). 2009; 28(6):1677-87. [PubMed: 19887408]

5. Cohen S, Van Handel M, Branson B, et al. Vital signs: HIV prevention through care and treatmentUnited States. MMWR Morb Mortal Wkly Rep. 2011; 60(47):1618-23. [PubMed: 22129997]

6. Bradley H, Hall HI, Wolitski RJ, et al. Vital Signs: HIV diagnosis, care, and treatment among persons living with HIV-United States, 2011. MMWR Morb Mortal Wkly Rep. 2014; 63(47):11137. [PubMed: 25426654]

7. Powers KA, Miller WC. Building on the HIV cascade: a complementary "HIV States and Transitions" framework for describing HIV diagnosis, care, and treatment at the population level. J Acquir Immune Defic Syndr. 2015; 69:341-7. [PubMed: 25835604]

8. Valdiserri RO, Forsyth AD, Yakovchenko V, et al. Measuring what matters: development of standard HIV core indicators across the U.S. Department of Health and Human Services. Public Health Rep. 2013; 128(5):354-9. [PubMed: 23997280]

9. National HIV/AIDS Strategy for the United States. Washington Office of National AIDS Policy (US). 2010

10. Ford, MA., Spicer, CM., editors. Monitoring HIV Care in the United States: Indicators and Data Systems. Washington DC: National Academy of Sciences; 2012.

11. Gunthard HF, Aberg JA, Eron JJ, et al. Antiretroviral treatment of adult HIV infection: 2014 recommendations of the International Antiviral Society-USA Panel. JAMA. 2014; 312(4):410-25. [PubMed: 25038359]

12. Lesko CR, Sampson LA, Miller WC, et al. Measuring the HIV care continuum using public health surveillance data in the United States. J Acquir Immune Defic Syndr. 2015; 70:489-94. [PubMed: 26258570]

13. Rebeiro PF, Althoff KN, Lau B, et al. Laboratory Measures as Proxies for Primary Care Encounters: Implications for Quantifying Clinical Retention Among HIV-Infected Adults in North America. Am J Epidemiol. 2015; 182(11):952-60. [PubMed: 26578717]

14. Gunthard HF, Saag MS, Benson CA, et al. Antiretroviral Drugs for Treatment and Prevention of HIV Infection in Adults: 2016 Recommendations of the International Antiviral Society-USA Panel. JAMA. 2016; 316(2):191-210. [PubMed: 27404187]

15. Nagin DS, Tremblay RE. Analyzing developmental trajectories of distinct but related behaviors: a group-based method. Psychol Methods. 2001; 6(1):18-34. [PubMed: 11285809]

16. Cote SM, Boivin M, Liu X, et al. Depression and anxiety symptoms: onset, developmental course and risk factors during early childhood. J Child Psychol Psychiatry. 2009; 50(10):1201-8. [PubMed: 19519755]

17. Hu MC, Muthen B, Schaffran C, et al. Developmental trajectories of criteria of nicotine dependence in adolescence. Drug Alcohol Depend. 2008; 98(1-2):94-104. [PubMed: 18602225]

18. van der Straten A, Shiboski S, Montgomery ET, et al. Patterns and predictors of adherence to diaphragm use in a phase III trial in sub-Saharan Africa: a trajectory analysis. J Acquir Immune Defic Syndr. 2009; 50(4):419-26. [PubMed: 19214115]

19. Nagin, DS. Group-based Trajectory Modeling of Development. Cambridge, MA: Harvard University Press; 2005.

20. Rebeiro P, Althoff KN, Buchacz K, et al. Retention among North American HIV-infected persons in clinical care, 2000-2008. J Acquir Immune Defic Syndr. 2013; 62(3):356-62. [PubMed: 23242158]

21. Yehia BR, Rebeiro P, Althoff KN, et al. Impact of age on retention in care and viral suppression. $\mathbf{J}$ Acquir Immune Defic Syndr. 2015; 68(4):413-9. [PubMed: 25559604]

22. Paz-Bailey G, Pham H, Oster AM, et al. Engagement in HIV care among HIV-positive men who have sex with men from 21 cities in the United States. AIDS Behav. 2014; 18(S3):348-58. [PubMed: 24026502]

23. Muthulingam D, Chin J, Hsu L, et al. Disparities in engagement in care and viral suppression among persons with HIV. J Acquir Immune Defic Syndr. 2013; 63(1):112-9. [PubMed: 23392459]

24. Miller WC, Lesko CR, Powers KA. The HIV care cascade: simple concept, complex realization. Sex Transm Dis. 2014; 41(1):41-2. [PubMed: 24326580] 
25. Buskin SE, Kent JB, Dombrowski JC, et al. Migration distorts surveillance estimates of engagement in care: results of public health investigations of persons who appear to be out of HIV care. Sex Transm Dis. 2014; 41(1):35-40. [PubMed: 24326579]

26. Nagin DS, Pagani L, Tremblay RE, et al. Life course turning points: the effect of grade retention on physical aggression. Dev Psychopathol. 2003; 15(2):343-61. [PubMed: 12931832] 


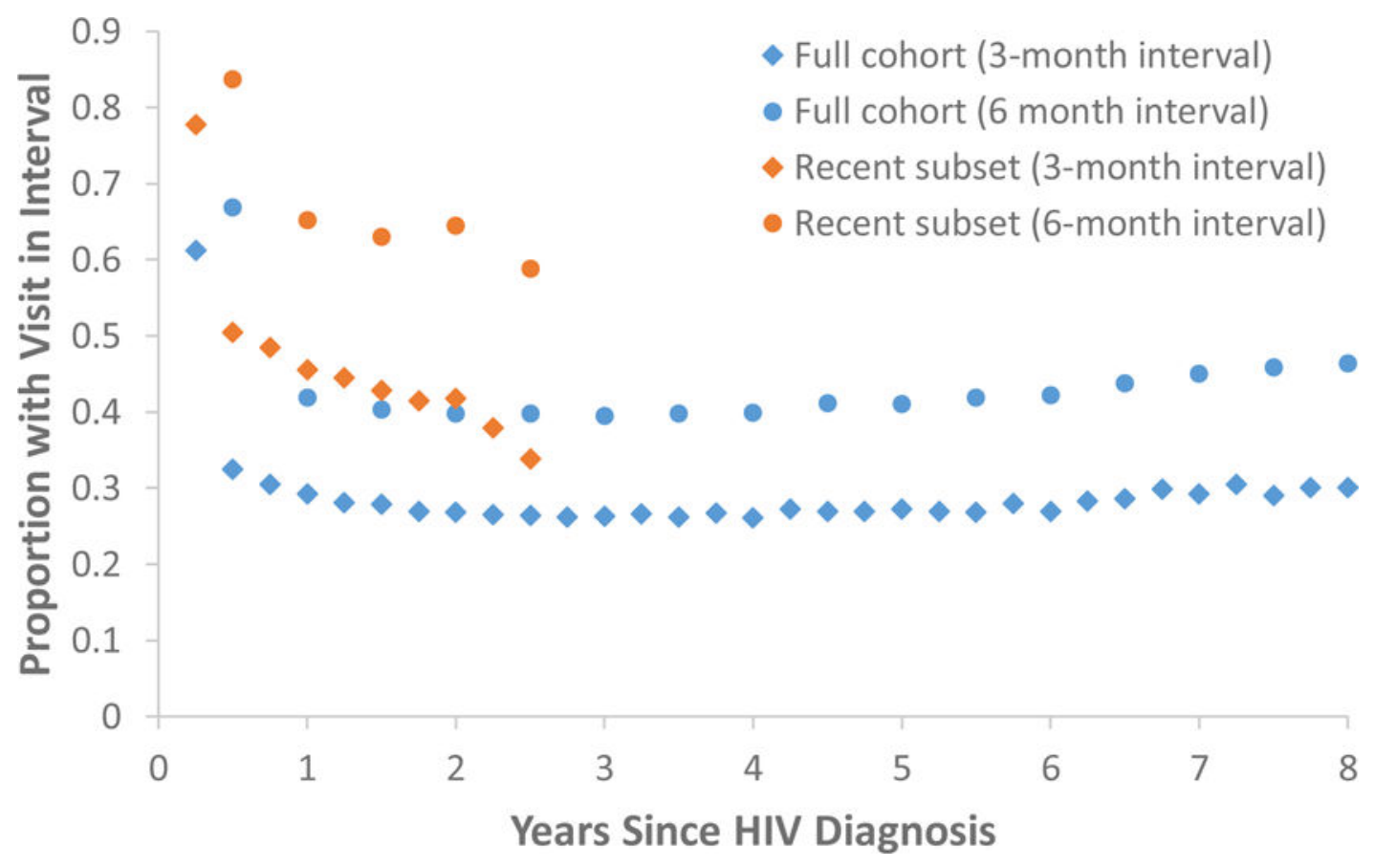

Figure 1. Overall HIV Care Attendance by Newly HIV-diagnosed Persons, North Carolina, 2006-2015

Observed proportions of newly HIV-diagnosed persons with CD4 or viral load results reported to surveillance in each three-month (diamonds) and six-month (circles) interval after diagnosis. Results are shown separately for the full cohort of persons diagnosed between March 31, 2006 and March 31, 2015 (blue) and the subset of persons diagnosed between July 1, 2013 and March 31, 2015 (orange). 

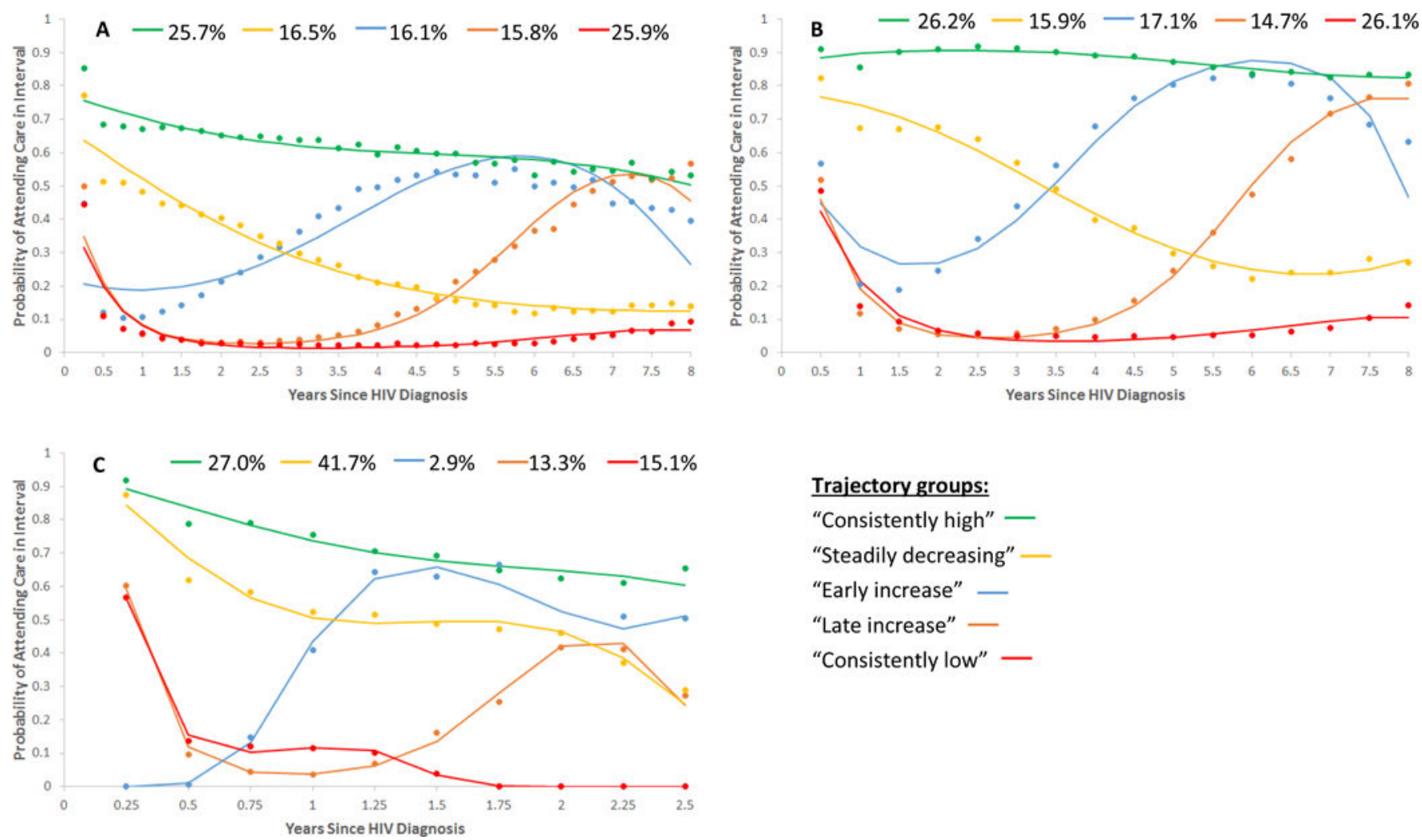

Figure 2. Longitudinal HIV Care Trajectories among Newly HIV-diagnosed Persons in North Carolina, 2006-2015

Predicted trajectories for the five-trajectory predictor-free model estimated in (A) the full cohort on the basis of three-month intervals, (B) the full cohort on the basis of six-month intervals, and $(\mathbf{C})$ the subset of more recent diagnoses on the basis of three-month intervals. Points represent the observed percentage of persons attending a visit in a given interval among those assigned to a given trajectory group on the basis of their maximum posterior group membership probability. Curves represent the proportion attending care over time as estimated by the model for a given trajectory group.

Note: Percentages shown at the top of each graph represent the predicted proportions of the cohort (or subset) following each trajectory. Also note the difference in the time axis scale for the population subset trajectories shown in Figure $2 \mathrm{C}$ vs. those in Figures $2 \mathrm{~A}$ and $2 \mathrm{~B}$. 


\section{Table 1}

Characteristics of Study Population

\begin{tabular}{|c|c|c|c|}
\hline & $\begin{array}{l}\text { Full cohort, 3-month outcome } \\
\qquad(\mathrm{N}=15887)\end{array}$ & $\begin{array}{l}\text { Full cohort, 6-month outcome } \\
\qquad(\mathrm{N}=15784)\end{array}$ & $\begin{array}{l}\text { Subset }{ }^{*}, \text { 3-month outcome } \\
(\mathrm{N}=\mathbf{2 4 5 0})\end{array}$ \\
\hline Median age at diagnosis (IQR) & $33.9(24.6,44.8)$ & $33.9(24.6,44.8)$ & $30.8(23.6,43.6)$ \\
\hline $\mathrm{N}(\%)$ male & 12079 (76.03) & $12004(76.05)$ & $1954(79.76)$ \\
\hline \multicolumn{4}{|l|}{ Race/ethnicity $[\mathrm{N}(\%)]$} \\
\hline Black, non-Hispanic & $10048(63.25)$ & $9974(63.19)$ & $1518(61.96)$ \\
\hline White, non-Hispanic & $3947(24.85)$ & $3923(24.85)$ & $605(24.7)$ \\
\hline Hispanic, any race & $1275(8.03)$ & $1270(8.05)$ & $219(8.94)$ \\
\hline Asian & $113(0.71)$ & $113(0.72)$ & $31(1.27)$ \\
\hline American Indian/Alaska Native & $91(0.57)$ & $91(0.58)$ & $22(0.90)$ \\
\hline Native Hawaiian/Other Pacific Islander & $13(0.08)$ & $13(0.08)$ & $2(0.08)$ \\
\hline Multi-race, non-Hispanic & $395(2.49)$ & $395(2.50)$ & $53(2.16)$ \\
\hline Unknown & $5(0.03)$ & $5(0.03)$ & $0(0.0)$ \\
\hline \multicolumn{4}{|l|}{ Risk group [N (\%)] } \\
\hline MSM only & $7109(44.75)$ & $7080(44.86)$ & $1266(51.7)$ \\
\hline IDU only & $290(1.83)$ & $288(1.82)$ & $34(1.39)$ \\
\hline Heterosexual contact only & $1866(11.75)$ & $1856(11.76)$ & $243(9.92)$ \\
\hline Multiple exposure types & $572(3.60)$ & $569(3.61)$ & $89(3.64)$ \\
\hline Other & $77(0.49)$ & $77(0.49)$ & $11(0.45)$ \\
\hline None reported or identified & $5973(37.59)$ & $5914(37.47)$ & $807(32.93)$ \\
\hline \multicolumn{4}{|l|}{ Years of follow-up [N (\%)] } \\
\hline$\geq 1$ & $15651(98.5)$ & $15651(99.2)$ & 2435 (99.4) \\
\hline$\geq 2$ & $14178(89.2)$ & $14178(89.8)$ & $1127(46.0)$ \\
\hline 23 & $12637(79.5)$ & $12637(80.1)$ & NA \\
\hline$\geq 4$ & $11140(70.1)$ & $11140(70.6)$ & NA \\
\hline 25 & $9488(59.7)$ & $9488(60.1)$ & NA \\
\hline 26 & $7812(49.2)$ & $7812(49.5)$ & NA \\
\hline$\geq 7$ & $6010(37.8)$ & $6010(38.1)$ & NA \\
\hline 28 & $4089(25.7)$ & 4089 (25.9) & NA \\
\hline$\geq 9$ & $2049(12.9)$ & $2049(13.0)$ & NA \\
\hline
\end{tabular}

Persons diagnosed between July 1, 2013 and March 31, 2015. 


\section{Table 2}

Odds Ratios (95\% Confidence Intervals) Associated with Predictors of Membership in Sub-Optimal Trajectory Groups

\begin{tabular}{|c|c|c|c|c|}
\hline \multicolumn{5}{|c|}{ Full cohort, three-month intervals } \\
\hline & Early increasing* & Late increasing* & Consistently low* & Steadily declining* \\
\hline Age at diagnosis ${ }^{\dagger}$ & $0.88(0.85,0.90)$ & $0.86(0.84,0.89)$ & $0.82(0.80,0.85)$ & $0.83(0.80,0.86)$ \\
\hline Male sex & $0.99(0.82,1.20)$ & $1.07(0.89,1.28)$ & $1.30(1.11,1.53)$ & $1.03(0.84,1.26)$ \\
\hline MSM only & $0.89(0.75,1.06)$ & $0.78(0.66,0.92)$ & $0.63(0.55,0.73)$ & $0.83(0.69,0.99)$ \\
\hline White non-Hispanic & $0.90(0.76,1.06)$ & $0.93(0.78,1.09)$ & $1.16(1.01,1.34)$ & $1.36(1.15,1.62)$ \\
\hline CD4 category ${ }^{t}$ & $0.83(0.79,0.88)$ & $0.87(0.83,0.92)$ & $0.99(0.94,1.04)$ & $0.91(0.86,0.96)$ \\
\hline \multicolumn{5}{|c|}{ Full cohort, six-month intervals } \\
\hline & Early increasing * & Late increasing $*$ & Consistently low ${ }^{*}$ & Steadily declining ${ }^{*}$ \\
\hline Age at diagnosis ${ }^{\dagger}$ & $0.88(0.86,0.91)$ & $0.88(0.85,0.91)$ & $0.81(0.78,0.83)$ & $0.82(0.79,0.85)$ \\
\hline Male sex & $1.02(0.85,1.22)$ & $1.02(0.84,1.25)$ & $1.38(1.17,1.64)$ & $1.01(0.81,1.25)$ \\
\hline MSM only & $0.91(0.77,1.07)$ & $0.74(0.62,0.90)$ & $0.60(0.51,0.69)$ & $0.76(0.62,0.92)$ \\
\hline White non-Hispanic & $0.92(0.79,1.07)$ & $0.84(0.70,1.02)$ & $1.17(1.02,1.36)$ & $1.18(0.98,1.42)$ \\
\hline CD4 category $t^{t}$ & $0.91(0.87,0.96)$ & $0.88(0.83,0.94)$ & $1.08(1.02,1.13)$ & $0.95(0.90,1.02)$ \\
\hline \multicolumn{5}{|c|}{ * vs. membership in "consistently high" group } \\
\hline
\end{tabular}

